\title{
Aproximación inicial a la comparación de cámaras hiperespectrales para su aplicación en agricultura
}

Initial approach to the comparison of hyperspectral cameras for application in agriculture

\section{Rodolfo José Piedra Camacho}

Piedra-Camacho, R. Aproximación inicial a la comparación de cámaras hiperespectrales para su aplicación en agricultura. Tecnología en marcha. Edición especial Movilidad Estudiantil 7. Abril, 2020. Pág.82-91.

doi) https://doi.org/10.18845/tm.v33i6.5170 


\title{
Palabras clave
}

Cámaras hiperespectrales; Agricultura; cubos de imágenes.

\section{Resumen}

El presente artículo muestra un método inicial, práctico y básico para la comparación de cámaras hiperespectrales. Una cámara hiperespectral permite tomar "cubos de imágenes" que constan de tres dimensiones, dos espaciales y una espectral en la que se almacena la reflexión de una escena a todos los espectros de onda entre el rango de la luz visible y el inicio del infrarrojo. Se utilizaron dos cámaras hiperespectrales para las pruebas, una marca Cubert modelo UHD185 y una cámara diseñada por el Grupo Integrado de Ingeniería de la Universidad de la Coruña; la primera captura 127 espectros en el rango de los 450 a 998nm, la segunda toma hasta 1088 espectros, algunos repetidos, en el rango de 385 - 950nm. Se diseñó una interfaz para el control de la cámara no comercial, se realizaron capturas de forma simultánea a una escena bajo abundante luz solar y se comparó la información mediante Matlab al promediar y presentar de forma gráfica la información hiperespectral de un área común a ambas capturas. Entre los resultados más predominantes se encuentra el reconocimiento de la suavidad en la curva de datos de la cámara UHD-185 y la falta de picos pronunciados en momentos de caída de nivel de ciertos espectros; ambos criterios de selección importante según la aplicación. El procedimiento se puede aplicar para cámara de línea o de escena siempre y cuando las mismas retornen su información en una gama de imágenes que se puedan pasar a un formato mat de Matlab.

\section{Keywords}

Hyperspectral cameras; Agriculture; cubes of images.

\begin{abstract}
This article shows an initial, practical and basic method for comparing hyperspectral cameras. A hyperspectral camera allows taking "image cubes" that consist of three dimensions, two spatial and one spectral in which the reflection of a scene is stored at all wave spectra between the range of visible light and the start of infrared. Two hyperspectral cameras were used for the tests, a Cubert brand model UHD-185 and a camera designed by the Integrated Engineering Group of the University of La Coruña; the first captures 127 spectra in the 450 to 998nm range, the second takes up to 1088 spectra, some repeated, in the $385-950 \mathrm{~nm}$ range. An interface was designed to control the non-commercial camera, captures were made simultaneously with a scene in abundant sunlight, and the information was compared using Matlab by averaging and graphically presenting hyperspectral information from an area common to both captures. . Among the most predominant results are the recognition of smoothness in the data curve of the UHD-185 camera and the lack of pronounced peaks in moments of level drop of certain spectra; both important selection criteria depending on the application. The procedure can be applied for line or scene cameras as long as they return their information in a range of images that can be passed to a Matlab .mat format.
\end{abstract}




\section{Introducción}

El avance y desarrollo de tecnologías de monitorización, vuelo a baja altura, sistemas de visión y manipulación de datos, ha llevado a la necesidad de la tecnificación de una variedad de industrias y áreas de la ciencia; de estas se destaca particularmente la agricultura, en principio por su importancia a nivel alimenticio. Particularmente la industria agrícola posee los medios con los cuales actuar sobre el medio, herbicidas, rotación de cultivos o similares; para complementar estos medios la tecnificación de la industria se da sobre el área de medición, monitorización y similares. Dentro de las tecnologías para la monitorización en agricultura destacan las imágenes, cámaras y análisis hiperespectrales.

Una imagen hiperespectral es una imagen que almacena la radiación de una gran cantidad de bandas espectrales que emiten los cuerpos terrestres al reflejar la energía del sol u otra emisión de luz [1]. Se puede comparar con el concepto de imagen RGB, donde cada imagen consta de tres espectros amplios: Rojo, azul y verde; una imagen hiperespectral consta de una mayor cantidad de espectros estrechos, principalmente del espectro visible y el infrarrojo; por la cantidad de espectros la imagen hiperespectral también se le conoce como cubo hiperespectral.

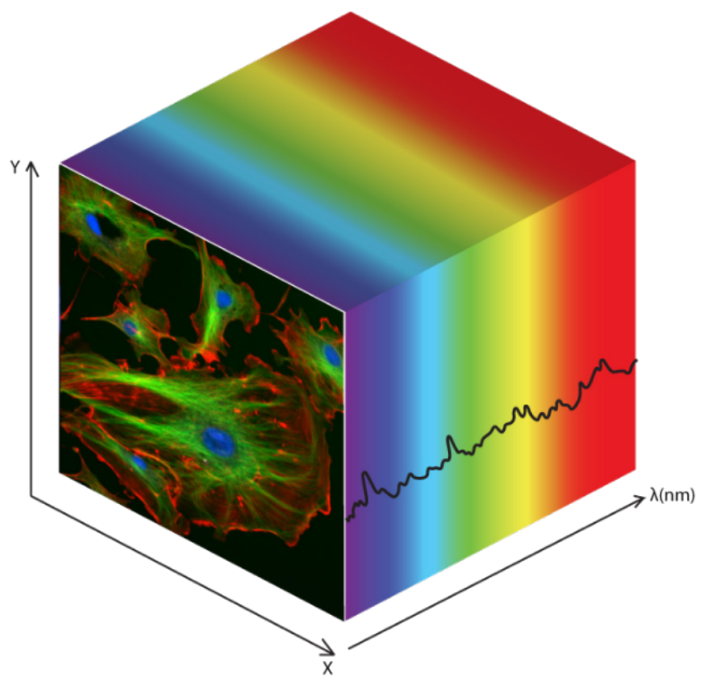

Figura 1. Concepto de Cubo Hiperespectral.

La utilidad del cubo hiperespectral radica en el concepto de firma espectral, lo cual es la forma específica en que cada material interactúa con las distintas longitudes de onda [2]. Entiéndase la firma espectral como una huella dactilar para un material que en el caso de la agricultura permite conocer sobre el estado del suelo, la biomasa o similares [2].

Finalmente como lo explica [1] existen tres tipos de cámaras hiperespectrales: de punto, de línea o push-broom y de escena o snapshot. En el primer tipo se captura la información en un único pixel, en el segundo se captura la información espectral en una línea de pixeles y el último captura información hiperespectral en toda la escena al costo de menos bandas espectrales.

Distintas investigaciones han demostrado la viabilidad de la tecnología, por un lado [3] utilizando una cámara snapshot, modelo UHD-185, obtuvieron información sobre la biomasa verde y el nivel de clorofila de una campo de cebada, requirió del algoritmo de vecino más cercano para interpretar la información hiperespectral limitada de la cámara UHD-185, imágenes de 50 × 50 pixeles. 
Por otro lado [4] realizaron un estudio con cámaras tipo pushbroom marca Headwall en el cual logran obtener información sobre la clorofila tipo a y b, así como el índice de área de hoja y la biomasa verde de los cultivos. En la misma línea [5] y [6] realizan más estudios mediante cámaras pushbroom variando principalmente en temas de cultivos o condiciones de la captura de información; no obstante, ambos demuestran la viabilidad del análisis hiperespectral.

En todos los casos se demuestra la viabilidad de las imágenes y cámaras hiperespectrales en el análisis de agricultura más no así el proceso o razonamiento bajo el cual se seleccionó o prefirió un modelo sobre otro; la selección en si misma puede incidir en la calidad de la información, detalle en espectros particulares, costos y facilidad de análisis; una cámara podría ser más rápida en la captura de imágenes pero incapaz de obtener el detalle en ciertos espectros críticos; si bien el detalle o calidad de la información varia de aplicación a aplicación si es cierto que siempre se necesita un proceso de elección. Por esta razón el presente artículo presenta un proceso básico e inicial bajo el cual se comparan dos cámaras hiperespectrales distintas, el estudio se realiza con capturas en tierra.

\section{Metodología}

\section{Equipo y Materiales}

Propiamente el proceso de comparación requirió de dos cámaras hiperespectrales, un trípode y dos computadores portátiles, una para el control de cada hiperespectrometro.

La primera cámara hiperespectral es del tipo snapshot, modelo UHD-185 de marca Cubert, captura imágenes con una resolución 50 × 50 en las imágenes espectrales y una resolución 1000 x 1000 en las imágenes blanco y negro; internamente la cámara traduce la información hiperespectral 50 × 50 a la imagen de mayor resolución; el rango espectral es de 450 a 998 nm y toma 127 espectros distintos con una distribución variable de $4 \mathrm{~nm}$ en los espectros bajos y de hasta $32 \mathrm{~nm}$ en los espectros altos. Posee un tiempo de exposición ajustable entre 1 a 1000 ms, para efectos de las pruebas se utilizó a 16 ms. Finalmente permite aplicar correcciones a las imágenes o entregar los datos crudos; para efectos de la pruebas se utilizó el método de captura de datos crudos.

La segunda cámara hiperespectral es del tipo pushbroom y fue diseñada propiamente por el Grupo Integrado de Ingeniería de la Universidad de la Coruña, campus Ferrol, utiliza una cámara UI3360-CP para la captura de imágenes. Al ser del tipo pushbroom las imágenes constan de una dimensión espacial, fijada a la dimensión $X$ y la información de todos los espectros en la dimensión Y, posee una resolución espacial de 2048 pixeles y toma 1088 bandas espectrales, de las cuales algunas se repiten, en el rango de 385 - 950nm. Requiere un tiempo de exposición mínimo de 83 ms, para efectos de las pruebas se utilizó el valor de 83 ms.

Se utilizó un trípode marca Nexstar para poder capturar una escena con la cámara tipo pushbroom, esto dado que al tomar solamente una línea espacial la cámara pushbroom requiere que su posición se ajuste recurrentemente para así poder capturar una escena entera; otra opción es que el trípode rote de forma continua en lo que la cámara hiperespectral toma las imágenes. Los trípodes marca Nexstar permiten ambas opciones de barrido.

Las computadoras portátiles se utilizaron para poder correr los programas necesarios para la captura de imágenes, en el caso de la cámara snapshot se utilizó el programa del proveedor Cubert de nombre Cube-Pilot; por otro lado el computador de la cámara pushbroom solo necesita ser de sistema operativo Windows y poder ejecutar programas diseñados en Microsoft Visual Studio 2012. 


\section{Interfaz de Control Pushbroom}

Para operar la cámara hiperespectral se diseñó un programa en $\mathrm{C}++$ bajo el ambiente de depuración Microsoft Visual Studio 2012 y utilizando como base las librerías del proveedor de la cámara UI3360-CP. En la figura 2 se puede apreciar la interfaz diseñada.

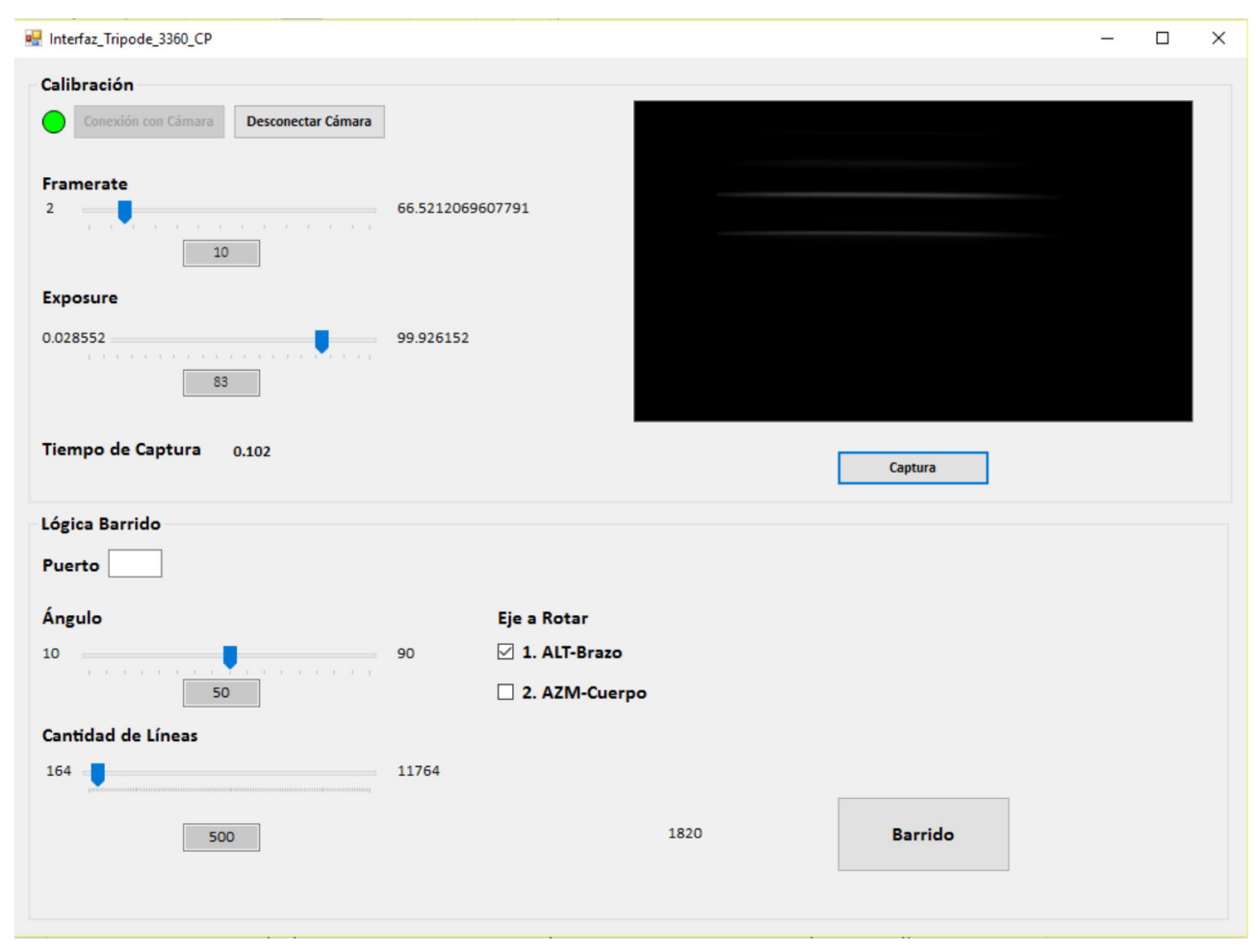

Figura 2. Interfaz para Captura de Imagenes Mediante Cámara Snapshot.

La interfaz permite controlar tanto la cámara como el trípode; propiamente permite asignar el tiempo de exposición, el ángulo del barrido, la cantidad de líneas espaciales a capturar, entre otros datos. Para el proceso de barrido del trípode, se decidió optar por un movimiento a velocidad constante, la velocidad depende de la siguiente fórmula:

$$
V \%=\frac{\text { angulo }^{\circ}}{T_{\text {captura }} S^{*} C_{\text {Lineas }}}
$$

\section{Condiciones y Procedimiento de Captura}

Las condiciones de la cámara pushbroom fueron las siguientes:

- Tiempo de Exposición = $83 \mathrm{~ms}$

- $\quad$ Ángulo $=20^{\circ}$

- $\quad$ Cantidad de Líneas $=350$

- Tiempo de Captura $=0.102 \mathrm{~s}$

- Velocidad $=0.56 \%$ 
Para la cámara tipo snapshot se utilizó la interfaz del proveedor Cube-Pilot sin aplicar corrección a las imágenes, tipo de captura por datos crudos, con una exposición de 16 ms y exportando los resultados a un formato .tiff.

Se tomaron las imágenes de forma simultánea a un área común, como la captura por cámara pushbroom toma más tiempo que la snapshot se sincronizó la captura de la cámara snapshot al punto intermedio del barrido; esto velando por que las condiciones de luz fuesen lo más similares en ambas capturas. Se realizaron las pruebas bajo techo; no obstante, la captura fue a un escenario en el exterior iluminado con abundante luz solar, no hubo obstrucciones mayores ni ventanas entre las cámaras y el escenario.

\section{Procesamiento en Matlab}

Para la comparación de los cubos de datos resultantes se utilizaron scripts .m de Matlab, propiamente los scripts realizan las labores:

- Generar el cubo de datos de las imágenes snapshot: La cámara retorna 127 imágenes .tiff separadas, un script .m se encarga de reunirlas en una estructura mat.

- Generar el cubo de datos de las imágenes pushbroom: Mismo proceso al de las imágenes snapshot.

- Comparación de sectores: Un script .m encargado de comparar dos áreas específicas y presentar a manera de gráfica la información promediada de la reflexión de espectros proveniente de cada cámara hiperespectral. Para las pruebas se comparó un sector del tronco del árbol presenten en la escena.

La salida final del procesamiento de Matlab es una gráfica donde se muestran todos los valores de cada espectro para cada cámara.

\section{Resultados y Discusión}

Los resultados de las pruebas se pueden resumir en tres imágenes, la captura por parte de cada cámara al área común y la gráfica de comparación. Inicialmente la figura 3 presenta la captura de la cámara pushbroom. 


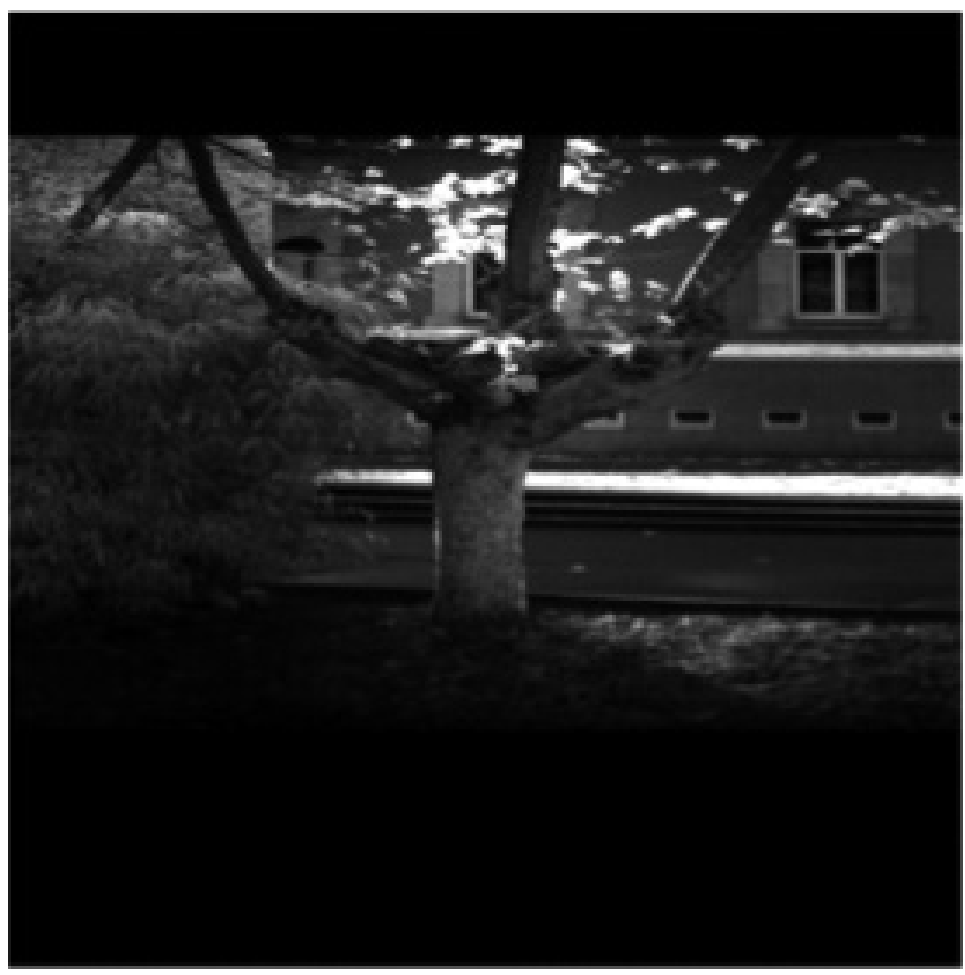

Figura 3. Imagen Cámara Pushbroom

Al provenir de un barrido de solo 350 líneas la imagen capturada no representa las dimensiones originales de la escena; por este efecto se aplicó una transformación de la dimensión X a 2048 líneas, logrando así una imagen cuadrada, este proceso se realizó solo para la presentación de la imagen, la comparación utiliza los datos originales de la matriz 2048 x 350. Las franjas completamente negras corresponden a un sector del sensor de la cámara UI3360-CP al cual no incide luz, como el área seleccionada para la comparación fue el tronco, esta particularidad no tiene mayor impacto.

El único detalle a destacar es que para aplicaciones formales en agricultura, conviene utilizar una cámara solidaria capaz de capturar imágenes generales de la escena, esto con el fin de localizar adecuadamente los sectores de la captura hiperespectral; esto aplica principalmente cuando la lejanía entre la escena y la cámara es mayor, como lo es un vuelo de baja altura de $120 \mathrm{~m}$.

La figura 4 presenta uno de los espectros de la cámara snapshot. 


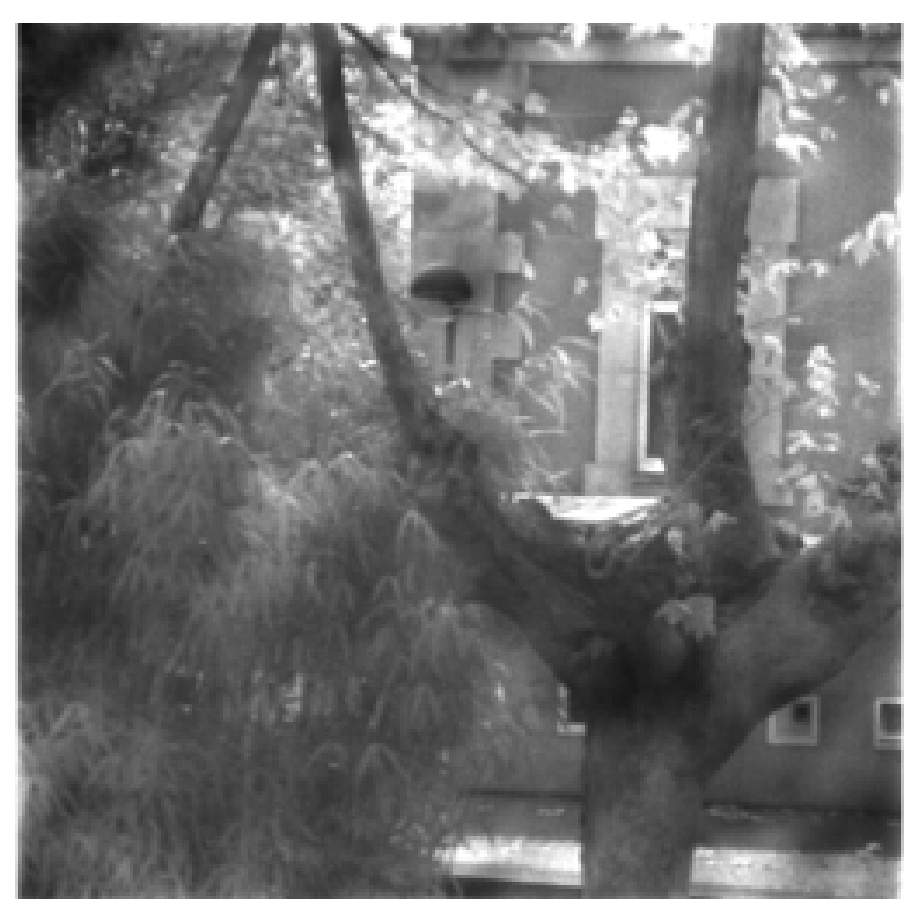

Figura 4. Imagen Cámara Snapshot.

La figura 4 presenta específicamente la reflexión al espectro de $746 \mathrm{~nm}$, por temas de presentación se aumentó el brillo de la imagen en un factor de 2.5; no obstante para la comparación se utilizó la información original de la captura.

Finalmente la figura 5 presenta el producto del script .m que compara la información hiperespectral del tronco de la escena.

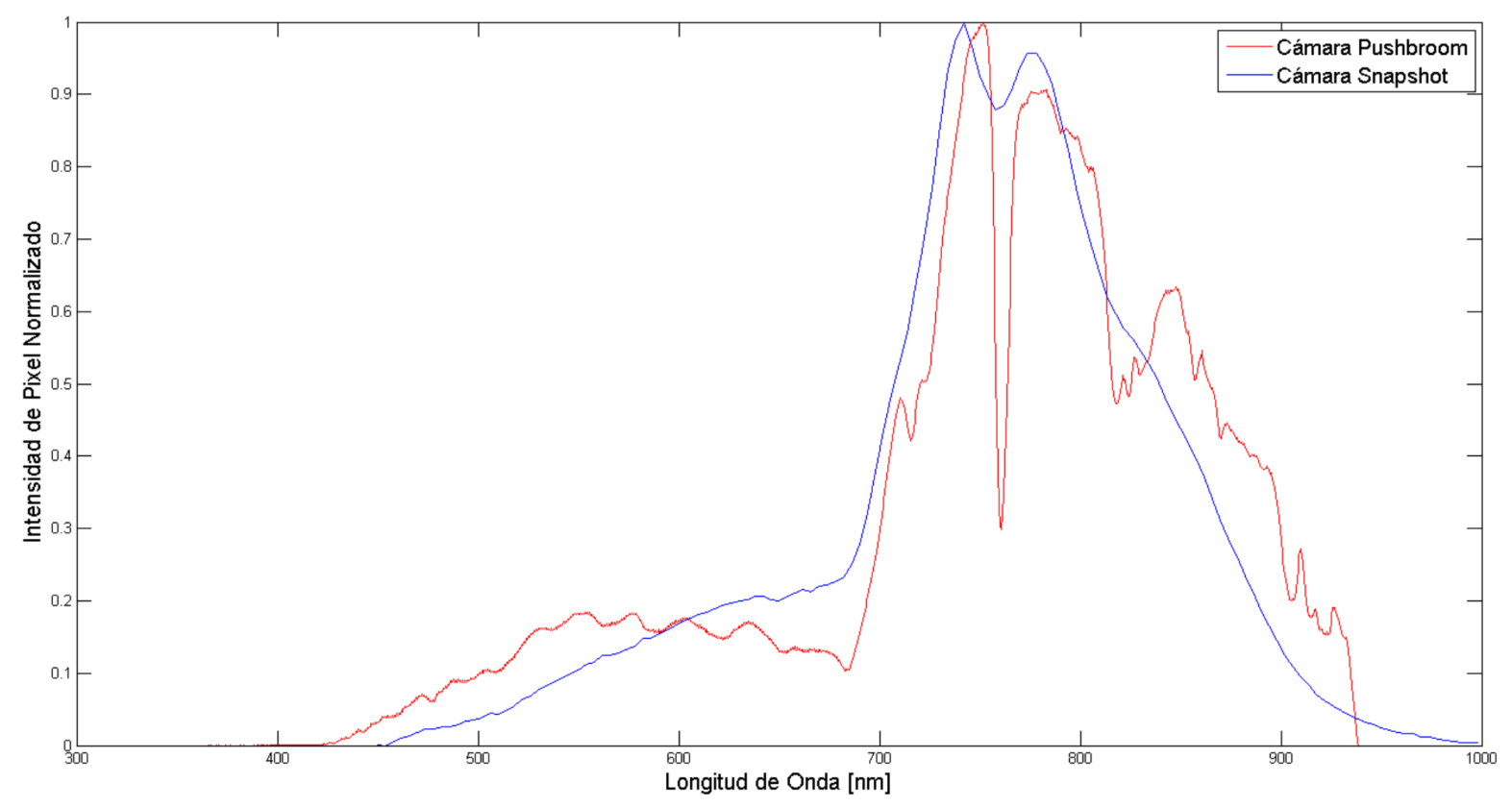

Figura 5. Comparación de Resultados de Cámaras Hiperespectrales. 
La información se normalizó para poder apreciar la información a una misma escala, por este motivo la principal comparación se debe apreciar en los perfiles de las gráficas y las proporciones internas de las mismas.

Cabe destacar que las cámaras hiperespectrales toman su información en el rango de la luz visible y el inicio del infrarrojo, dado que el primer espectro de luz visible responde al 400nm se puede verificar con la gráfica que ambas cámaras presentan un comportamiento inicialmente adecuado; por otro lado los valores máximos de 950 y 998nm son parte del primer sector del espectro infrarrojo por lo que las cámaras respetan adecuadamente el límite superior del espectro.

El desfase entre los perfiles de las gráficas se puede atribuir a temas de calibración por parte de la cámara tipo pushbroom; esta diferencia aunque importante de corregir no implica un defecto mayor que pueda ser criterio de elección o contraste, siempre y cuando sea posible calibrar el equipo.

Un primer problema que se observa con las gráficas es el detalle en la reflexión de los espectros por parte de la cámara snapshot, principalmente la progresión sin cambios pronunciados que es prueba de un proceso de suavizado de datos. En la gráfica de cámara pushbroom se puede observar como en el perfil de la gráfica existen zonas con cambios pronunciados; la falta de este fenómeno en la cámara snapshot es motivo de cuidado al seleccionar el equipo.

Seguidamente las gráficas hacen luz a un segundo problema, la caída pronunciada en la gráfica pushbroom entre los espectros de 700 y 800nm casi no tiene contraparte en la gráfica snapshot. Debe aclararse que existen espectros de la luz solar que son absorbidos por la atmósfera terrestre, la caída entre los 700 y 800nm de la cámara pushbroom reflejan este fenómeno efectivamente; por otro lado la cámara snapshot presenta únicamente un leve valle en el mismo rango espectral, por hipótesis se puede decir que este problema es una extensión del proceso de suavizado de datos realizado por la cámara UHD-185.

La importancia del problema radica en que parte de la información que interesa para el análisis de parámetros en agricultura se encuentran en el rango de 700 - 800nm o bien en la capacidad de presentar los cambios pronunciados; una medición suavizada o que carece de la reflexión de ciertos fenómenos atmosféricos y de humedad introduce un error a las mediciones de parámetros en agricultura. Cabe destacar que ambos problemas muestran una diferencia relevante en cuanto a la sensibilidad al detalle de las cámaras y son posible motivo para la elección de un equipo sobre el otro.

\section{Conclusiones}

El proceso de contraste realizado utilizó una gráfica para mostrar el promedio de la información hiperespectral de una porción de madera en una escena capturada tanto con una cámara pushbroom como una cámara snapshot modelo UHD-185. El proceso de comparación dio a luz dos principales diferencias o problemas.

La cámara snapshot presenta un suavizado de datos que elimina la sensibilidad a los cambios bruscos o los máximos en la reflexión de espectros, dejando por resultado una curva suave; esto es contrario a la cámara pushbroom utilizada que presenta la información incluyendo los cambios bruscos. Efectivamente una pérdida de información en la cámara snapshot.

La cámara snapshot no detecta debidamente la caída en el rango de los 700 a 800nm que responde a la absorción, por parte de la atmósfera, de ciertas longitudes de onda. Esto puede volver inutilizable una cierta parte de la información según la aplicación. 
En ambos casos cabe aclarar que la elección propia de un equipo dependerá únicamente de las necesidades de la aplicación y como las cámaras responden a dicha aplicación; en el caso de la agricultura esto puede variar según los parámetros que se quieran estudiar. Por otro lado siempre es posible tomar la información de comparación y decidir qué acciones tomar sobre las cámaras para poder utilizar su información, por ejemplo para el caso de la cámara snapshot utilizada tomar la información y tratar de extraer los cambios pronunciados originales.

De esta forma se puede concluir que el proceso de comparación, aunque básico y práctico permitió diferenciar dos cámaras hiperespectrales en criterios de importancia para elección, así mismo logro traer a luz necesidades de calibración y similares.

\section{Agradecimientos}

Todo el equipo y material en el desarrollo del proyecto se debe al Grupo Integrado de Ingeniería de la Universidad de la Coruña, Campus Ferrol; se agradece tanto el aporte material así como en conocimiento y experiencia de la materia o facilitación de documentación. Así mismo se agradece al sistema de Beca de Movilidad de Rectoría del TEC por brindar el aporte económico necesario para dar posibilidad al desarrollo del proyecto de graduación, el presente artículo es fruto y prueba del apoyo significativo de la beca.

\section{Referencias}

[1] B. Priego Torres (Julio de 2011). Técnicas de Inteligencia Computacional Aplicadas al Procesamiento de Señal en Imágenes Hiperespectrales. (Tesis de Maestría). Universidad de la Coruña, Coruña, España.

[2] A. Marini \& P. Colandrea (2009). Data Bank Of Territorial Spectral Signatures For Sardinia And North Africa. NATO Science for Peace and Security Series C: Environmental Security (págs. 137-142). Dordrecht: Springer.

[3] H. Aasen, A. Burkart, A. Bolten \& G. Bareth. "Generating 3D hyperspectral information with lightweight UAV snapshot cameras for vegetation monitoring: From camera calibration to quality assurance", ISPRS Journal of Photogrammetry and Remote Sensing, pp. 245-259, 2015.

[4] Z. Malenovský, T. Veness, L. \& Wallace, "HyperUAS-Imaging Spectroscopy from a Multirotor Unmanned Aircraft System", Journal of Field Robotics, pp. 571-590, 2014.

[5] J. Suomalainen, et al., "Lightweight Hyperespectral Mapping System and Photogrammetric Processing Chain for Unmanned Aerial Vehicles". Remote Sensing, pp. 11013-11030, 2014.

[6] P. Zarco-Tejeda, V. González-Dugo \& J. Berni, "Fluorescence, temperature and narrow-band indices acquired from a UAV platform for water stress detection using a micro-hyperspectral imager and a thermal camera". Remote Sensing of Environment, pp. 322-337, 2012. 